\title{
Sprechstundenhilfe in der Praxis - und auf Hausbesuch
}

Felix Schürch

Korrespondenz:

Dr. med. Felix Schürch

Albulastrasse 52

CH-8048 Zürich

felix.schurch[at]hin.ch

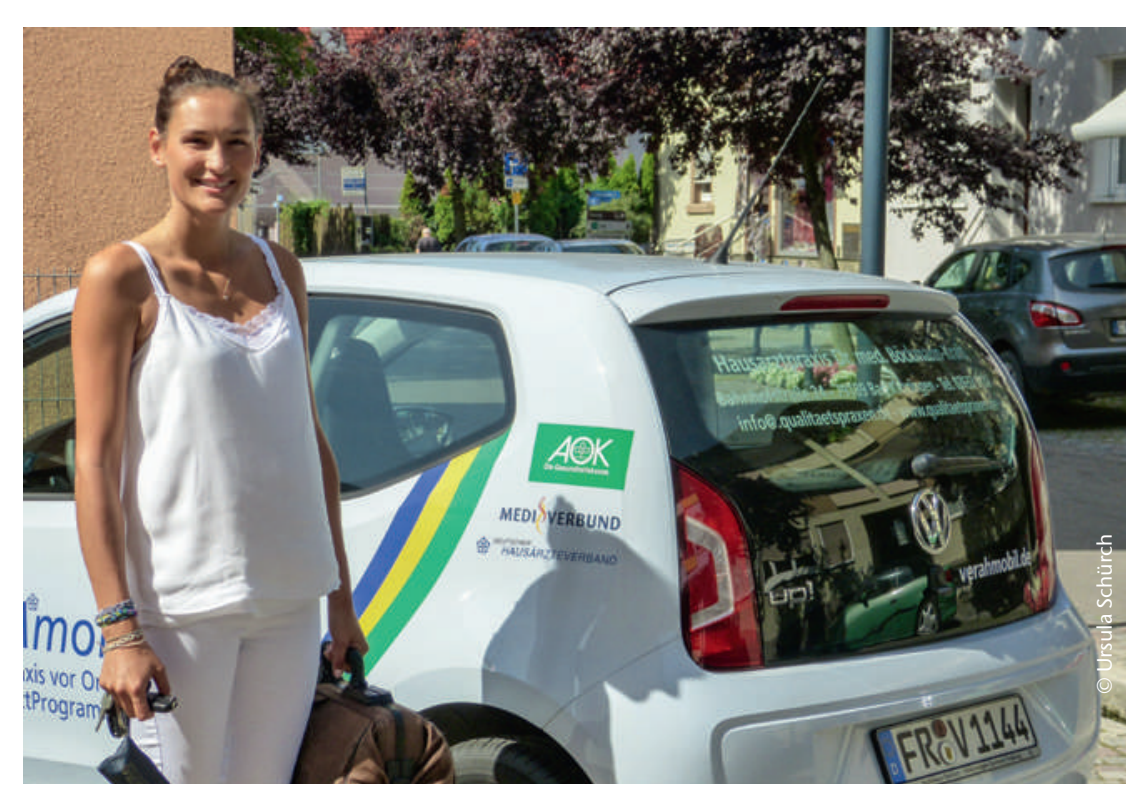

Mehrmals wöchentlich fährt die «Sprechstundenhilfe» Frau Link mit ihrem Dienstwagen auf

Hausbesuch zu chronisch-kranken Menschen mit eingeschränkter Mobilität.

Der Mangel an Hausärzten bei gleichzeitig steigender Anzahl an chronisch kranken Menschen ist in Deutschland genau so eine Tatsache wie in der Schweiz. Durch Fortbildungsmodule für die Mitarbeiterinnen in den Hausarztpraxen erlangen nichtakademische Praxisassistentinnen zusätzliche Kompetenzen und können zur Entlastung der Ärzte Hausbesuche machen. Dadurch kommen die Fähigkeiten dieser erfahrenen und empathischen Fachfrauen nutzbringend zur Anwendung, die Arbeit in der Hausarztpraxis wird für alle im Team vielfältiger, entspannter und abwechslungsreicher. Eines der am meisten verbreitenden Versorgungsmodelle in Deutschland heisst VERAH. Ein Augenschein in einer Hausarztpraxis bei Freiburg i. Br. zeigt, was konkret hinter diesem Konzept steckt.

Machen Sie Hausbesuche? Diese Frage hört man häufig bei einem ersten Kontakt mit einer neuen Patientin. Offenbar hat das Thema für die Patientinnen und Patienten eine hohe Priorität. Harro Böckmann ist Hausarzt in Bad Krozingen, einer Kleinstadt zwischen Freiburg und Basel. Er macht Hausbesuche. Bei Dr. Böckmann arbeitet Sabrina Link als Sprechstundenhilfe. Auch Frau Link macht Hausbesuche.

Sabrina Link ist von Berufes wegen «Sprechstundenhilfe» oder «Arzthelferin» - wenn man diese antiquierten Berufsbezeichnungen überhaupt noch benützen will. Richtig ist heute in Deutschland die Bezeichnung Medizinische Fachangestellte (MFA), in

\section{Les assistantes médicales \\ au cabinet - et à domicile}

L'Allemagne a mis en place des modules de formation continue à destination des assistantes médicales des cabinets de médecine de famille. En cours du soir ou en cours hebdomadaires, ces assistantes expérimentées ont ainsi la possibilité d'acquérir des compétences supplémentaires leur permettant de décharger le médecin de famille et de participer à la prise en charge des personnes atteintes de maladies chroniques. Lors de visites à domicile, elles peuvent également surveiller l'évolution d'une insuffisance cardiaque, mesurer le taux de sucre et la pression sanguine en cas de malaise, ou encore effectuer un contrôle de plaies suite à un séjour en clinique par exemple. Cette nouvelle qualification, dénommée Verah (pour assistantes de prise en charge des patients au cabinet médical), a déjà été obtenue par plus de 4000 personnes qui exercent maintenant dans toute l'Allemagne. Le médecin de famille et les «Verah» travaillent ensemble au cabinet médical et suivent souvent leurs patients depuis plusieurs années. Les Verah sont donc en mesure de garantir une continuité optimale dans la prise en charge à long terme des patients, sans augmentation significative de la charge administrative pour le médecin.

Österreich Arztassistentin und in der Schweiz Medizinische Praxisassistentin (MPA). Sabrina Link ist demnach eine Medizinische Fachangestellte - und zusätzlich ist sie eine Verah. Das Akronym VERAH steht für Versorgungsassistentin in der Hausarztpraxis. Mit der Zusatzausbildung zur Versorgungsassistentin hat Frau Link die Kompetenz und Fähigkeit erworben, im Auftrag des Arztes selbstständig Hausbesuche zu machen. Frau Link hat ein bis sechs Einsätze pro Woche, daneben ist sie als «gewöhnliche» MFA in der Doppelpraxis Böckmann/Kraft in Bad Krozingen tätig.

\section{Herzinsuffizienz, Schwindelattacken und Wundkontrollen}

Unmittelbar vor einem Hausbesuch wird aus der elektronischen Krankengeschichte ein «Laufzettel» mit 
